http://dx.doi.org/10.4314/jae.v15i2.5

\title{
Assessment of Climate Change Adaptive Strategies in Small Ruminant Production in Rural Nigeria
}

\author{
Tologbonse, E.B., Iyiola-Tunji, A.O., Issa, F.O., Jaliya, M.M., Daudu, C.K., \\ Adedokun, I.K. and Okoro, B.I. \\ National Agricultural Extension and Research Liaison Services, Ahmadu Bello University, Zaria \\ Corresponding author: deletologbonse@gmail.com ; deletologbonse@yahoo.com
}

\begin{abstract}
Animals are intrinsically dependent on the environment, and any fluctuations in weather and climate can affect them through water and land changes, such as desertification, feed and water availability. Climate change will not only impact the health and welfare of animals, but also the more than a billion people who depend on them. This study was therefore aimed at evaluating the adaptive measures used by rural farmers to alleviate the effect of climate change on small ruminant (sheep and goats) production in rural Nigeria. The target population for this study was all the small ruminant farmers in the five agro-ecological zones of Nigeria. A total of 300 respondents were interviewed using validated structured interview schedule. Data were analyzed using frequency counts, means and percentage. The result shows that most (71.9\%) of the respondents were adults between 20 and 50 years of age. About 35\% keep sheep and goats together while others keep either of the species. The most preferred management system is semi-extensive system (64\%). Majority (93.2\%) of small ruminant farmers in the rural Nigeria were aware of what climate change is all about and about $54 \%$ of them had observed changes in climage through personal experience.Majority of them had also observed and attributed changes to effect of climate change on their flock. Majority of them have also used various strategies to combat climate change. Traditional health care and irrigation of pasture during dry season were not effective adaptive strategies to combat climate change. There is need to educate the farmers more on the possible effect of climate change through the use of radio and other extension organizations (government and non-governmental) on the current situation and implication of climate change on themselves and their animals
\end{abstract}

Key words: Adaptive strategies climate change, small ruminant.

\section{Introduction}

Climate change is a major challenge to agricultural development in Africa and the world at large as reported by Technical Centre for Agricultural and Rural Co-operation (2009). It is not only a challenge to agricultural development but to food security and the general livelihoods conditions of any population. Agriculture, being one of the most 
weather-dependent of all human activities is highly vulnerable to climate change. African countries are particularly vulnerable to climate change because of their dependence on rain fed agriculture, high levels of poverty, low levels of human and physical capital, inequitable land distribution and poor infrastructure (Watson et al., 1997). Africa, like the rest of the world, is experiencing increasing risk from climate change including rising temperatures and heat waves, shortfalls in water supply/increasing floods arising from shortage/excessive rainfalls, sea level rise, increasing likelihood of conflict and induced environmental and vector borne diseases . These conditions emanating from climate change are bound to compromise agricultural production (crop, livestock, forest and fishery resources), nutritional and health statuses, trading in agricultural commodities, human settlements (especially of agricultural communities), tourism and recreation among others.

Generally climate change is expected to have a mixed effect on agriculture with some areas benefiting from moderate temperature increases and others being negatively affected. Positive effects of climate change could arise from changes in seasons and production cycles. For example, Ethiopia and Southern Africa are expected to have extended growing seasons as a consequence of increased temperature and rainfall. In the same vein, livestock production could be boosted by temperature increases (FAO, 2009). Conversely, Deressa and Hassan (2009) found increasing temperatures to be particularly damaging to Ethiopian agriculture, a situation that is not uniformly distributed across agro-ecological zones. Kurukulsuriya and Mendelson (2007) equally indicated that African agriculture is sensitive to climate change in the sense that farmers will experience net revenue losses from warming especially with reduction in precipitation. Also, climate change is thought to be responsible for conflict in Darfur where a combination of decades of drought, desertification and overpopulation are among the causes of conflict in that the Baggara Arab nomads search of water have to take their livestock further south, to lands mainly occupied by farming peoples ( IFPRI, 2009).

The above effects of climate change can be said to hold true for Nigeria in the sense that the same ecological conditions applies especially in the savanna and sahelian regions of the north. Technical Centre for Agricultural and Rural Co-operation (2009) observed that climate change has led to a reduction in livelihood options in many African, Carribean and Pacific (ACP) countries. For instance demographic shifts within rural societies, low level of education and poorly developed communication and market infrastructure is seen as complicating the task of developing location-specific response that effectively address the issue. It further highlighted the importance of facilitating communication among and between various actors to contribute to the effectiveness of intervention strategies. 
It is believed that adaptation strategies to combat the effects of climate change and also ensuring improve and sustainable livelihood for the farm family depends on the knowledge, attitudes, practices and belief systems of farmers. Animals are intrinsically dependent on the environment, and any fluctuations in weather and climate can affect them through water and land changes, such as desertification and feed and water availability. Climate change will not only impact the health and welfare of animals, but also the more than a billion people who depend on them. Thus this study aimed at evaluating the adaptive strategies used by rural farmers to alleviate the effect of climate change on small ruminant (sheep and goats). The general objective of the study was to assess climate change adaptive strategies in small ruminant production in rural Nigeria.

The specific objectives were to:

i. describe their production characteristics;

ii. ascertain small ruminant farmers understanding of climate change and sources of awareness;

iii. describe the climate change parameters observed by farmers;

iv. determine the frequency of observed changes in small ruminant due to climate change; and

v. ascertain the adaptive methods used by small ruminant farmers and the effectiveness.

\section{Methodology}

The target population for this study were all the small ruminant farmers in the five agroecological zones of Nigeria namely North-West, North-East, North-Central, South West and South-East. From each zone, the state where NAERLS zonal office and the coordinating research institute of the national agriculture research system is located was purposively selected for ease of administration and retrieval of questionnaire, namely, Kaduna, Borno, Niger, Oyo and Abia. In each of the selected states, two villages that were known to have high population of ruminants farmers were purposively selected.Thirty farmers were randomly selected from each village. In total, 300 respondents were interviewed. Validated structured interview schedule was used to obtain primary data from the rural small ruminant farmers with the assistance of trained enumerators that understood the local language. Data were analyzed using frequency counts, means and percentages. A 4-point Likert type scale of very effective, effective, fairly effective and not effective of which was assigned weights of 4,3,2,1, respectively was used to measure level of effectiveness of adaptive strategies adopted by respondents. In calculating perception of level of effectiveness of adaptive strategies adopted, the mid-point values of the scale were summed and further divided by 4 to obtain mean of 2.5. Any adaptive strategy with a mean score of equal or above the cutoff mean of 2.5 was regarded and perceived as effective and any mean score of lower than 2.5 was perceived as not effective. 


\section{Results and Discussion}

\section{Socio-economic characteristics of small ruminant farmers in rural Nigeria}

The socio-economic characteristics of small Ruminant Farmers In Rural Nigeria on state and agro-ecological basis is shown in Table 1. Sixty-six percent of the respondents were male. They were mostly in the active age range of between 20 and 50 years $(71.9 \%)$ and majority $(86.0 \%)$ were married. This corroborates the findings of Ajala; Lamidi and Otaru (2008) on the age range of small ruminant farmers in the Northern Guinea Savanna zone of Nigeria. About $80 \%$ of the respondents had between 10 and below house hold size. Farming was the major (55.3\%) primary occupation engaged in by the Nigerian rural small ruminant farmers. About $2 \%$ of the respondents were not employed in any other business, but still keep small ruminants. Majority $(42.3 \%)$ of the respondents were illiterate and had no education and about $31 \%$ had primary education.

Table 1: Socio-economic characteristics of small ruminant farmers in Rural Nigeria $(n=300)$

\begin{tabular}{|c|c|c|c|c|c|c|}
\hline \multirow[t]{2}{*}{ Variables } & \multicolumn{5}{|c|}{ State/Agro-ecological zone } & \multirow[t]{2}{*}{ Total } \\
\hline & $\begin{array}{l}\text { Kaduna- } \\
\text { North-west }\end{array}$ & $\begin{array}{l}\text { Niger- } \\
\text { North- } \\
\text { central }\end{array}$ & $\begin{array}{l}\text { Borno- } \\
\text { North-east }\end{array}$ & $\begin{array}{l}\text { Oyo- } \\
\text { South-west }\end{array}$ & $\begin{array}{l}\text { Abia- } \\
\text { Southeast }\end{array}$ & \\
\hline \multicolumn{7}{|l|}{ Sex } \\
\hline Male & $37(61.7)$ & 55 (91.7) & $46(76.7)$ & $31(51.7)$ & $29(48.3)$ & $\begin{array}{l}198 \\
(66.0)\end{array}$ \\
\hline Female & $23(38.3)$ & $5(8.3)$ & 14 (23.3) & $29(48.3)$ & $31(51.7)$ & $\begin{array}{l}102 \\
(34.0)\end{array}$ \\
\hline
\end{tabular}


Journal of Agricultural Extension

Vol. 15 (2), December, 2011

\section{Age (years)}

$20-30$

$31-40$

$41-50$

$51-60$

$61 \&$ above

\section{Marital Status}

single

married

Divorced

widowed

House hold size

5 \& below

6-10

$11-15$

$16 \&$ above

Educational level

none

Qranic

Adult education

Primary

Secondary

Post secondary
19 (31.7)

17 (28.3)

$11(18.3)$

$$
7 \text { (11.7) }
$$

$6(10.0)$

6 (10.0)

47 (78.3)

4 (6.7)

$3(5.0)$

19 (31.7)

12 (20.0)

$12(20.0)$

17 (28.3)

33 (55)

$0(0.0)$

$0(0.0)$

$12(20.0)$

$0(0.0)$

15 (25.0)
$18(30.0)$

17 (28.3)

$4(6.7)$

$6(10.0)$

$10(16.7)$

8 (13.3)

$13(21.7) \quad 23(38.3) \quad 17(28.3) \quad 15(25.0) \quad 79(26.3)$

$13(21.7) \quad 23(38.3) \quad 17(28.3) \quad 15(25.0) \quad 79(26.3)$

$1(1.7)$

$4(6.7)$

15 ( 25.0) $17(28.3)$

44

(14.7)

$0(0.0))$

$6(10.0)$

$14(23.3)$

$14(23.3)$

$40(13.3)$

5 (8.3)

$1(1.7)$

2 (3.3)

$0(0.0)$

$14(4.7)$

$54(90.0) \quad 53(88.3) \quad 51(85.0) \quad 53(88.3)$

(86.0)

7 (2.3)

$1(1.7)$

$4(6.7)$

$1(1.7)$

2 (3.3)

$6(10.0) \quad 5(8.3)$

16 (5.3)

2 (3.2) 


\section{Primary occupation}

\begin{tabular}{|c|c|c|c|c|c|c|}
\hline Civil servant & $6(10.0)$ & $9(15.0)$ & $4(6.7)$ & $3(5.0)$ & $4(6.7)$ & $26(8.7)$ \\
\hline Farming & $22(36.7)$ & $40(66.7)$ & $33(55.0)$ & 26 (43.3) & $45(75.0)$ & $\begin{array}{l}166 \\
(55.3)\end{array}$ \\
\hline $\begin{array}{l}\text { Unemployed } \\
\text { (student) }\end{array}$ & $3(5.0)$ & 2 (3.3) & $0(0.0)$ & $0(0.0)$ & $0(0.0)$ & $5(1.7)$ \\
\hline $\begin{array}{l}\text { Traders/artisans } \\
\text { others }\end{array}$ & 29 (48.3) & $9(15)$ & $23(38.3)$ & $31(51.6)$ & $11(18.3)$ & $\begin{array}{l}103 \\
(34.3)\end{array}$ \\
\hline
\end{tabular}

Source: Field survey, 2010; Figures in parenthesis are percentage

\section{Small ruminant production characteristics}

Table 2 shows the small ruminant production characteristics in rural Nigeria. The results shows that majority (50\%) of the farmers were rearing only goats and about $35 \%$ rearing and sheep and goats. Circumstances under which both species of small ruminants being reared together was also observed. Semi-intensive system was the most (64\%) prominent system of rearing sheep and goats in the study area. However, on agroecological basis, about $93 \%$ practiced intensive system of rearing sheep and goat in the South-East zone.

Table 2: Distribution of respondents by production characteristics in rural Nigeria $(n=300)$

\begin{tabular}{lllllll}
\hline Variables & \multicolumn{3}{l}{ State/Agro-ecological zone } & & Total \\
\cline { 2 - 6 } & $\begin{array}{l}\text { Kaduna- } \\
\text { North- } \\
\text { west }\end{array}$ & $\begin{array}{l}\text { Niger- } \\
\text { North- } \\
\text { central }\end{array}$ & $\begin{array}{l}\text { Borno- } \\
\text { North- } \\
\text { east }\end{array}$ & $\begin{array}{l}\text { Oyo- } \\
\text { South- } \\
\text { west }\end{array}$ & $\begin{array}{l}\text { Abia- } \\
\text { Southeast }\end{array}$ & \\
\hline Type of small ruminant kept & & & & & & \\
Sheep only & $18(30.0)$ & $12(20.0)$ & $18(30.0)$ & $1(1.7)$ & $0(0.0)$ & $49(16.3)$ \\
Goats only & $25(41.7)$ & $22(36.7)$ & $8(13.3)$ & $46(76.7)$ & $49(81.7)$ & $150(50.0)$ \\
Both sheep and goat & $17(28.3)$ & $26(43.3)$ & $34(56.7)$ & $13(21.7)$ & $11(18.3)$ & $104(34.7)$
\end{tabular}

Flock management system

Intensive

$4(6.7) \quad 13(21.7) \quad 14(23.3) \quad 0(0) \quad 56(93.3) \quad 87(29.0)$ 
Journal of Agricultural Extension

Vol. 15 (2), December, 2011

Semi-intensive

$46(76.6) \quad 46(76.6)$

$39(65.0)$

$57(95.0)$

$4(6.6)$

$192(64.0)$

extensive

$7(11.7)$

$3(5.0)$

$0(0.0)$

$21(7.0)$

\section{Number of sheep in flock/herd}

None

$1-5$

6-10

11-15

$16 \&$ above

\section{Number of Goat in flock/herd}

None

$1-5$

6-10

11-15

$16 \&$ above
17 (28.3) $22(36.7)$

$13(21.7) \quad 45(75.0)$

$47(78.3)$

144 (48.0)

4.2

$13(21.7) \quad 25(41.7)$

$26(43.3)$

8 (13.3)

$8(13.3)$

$80(26.7)$

$12(20.0)$

$10(16.7)$

$12(20.0) \quad 5(8.3)$

$3(5.0)$

$42(14)$

$4(6.6)$

5 (8.3)

$3(5.0)$

1(1.7)

$0(0)$

13(4.3)

$14(23.3) \quad 0(0.0)$

$6(10.0)$

$1(1.7)$

$0(0.0)$

$21(7.0)$

$\begin{array}{lllllll}23(38.3) & 12(20.3) & 22(36.7) & 2(3.3) & 0(0) & 59(19.7) & 6.5 \\ 8(13.3) & 22(37.3) & 14(23.3) & 15(25.0) & 30(50.0) & 89(29.8) & \\ 7(11.7) & 23(39.0) & 17(28.3) & 33(55.0) & 27(45.0) & 107(35.8) & \\ 9(15.0) & 2(3.4) & 3(5.0) & 9(15.0) & 1(1.7) & 24(8.0) \\ 13(21.7) & 0(0.0) & 4(6.7) & 1(1.7) & 2(3.3) & 20(6.7)\end{array}$

Source: Field survey, 2010; Figures in parenthesis are percentage 


\section{Farmers' understanding, observation and sources of awareness of climate change:}

Table 3 shows Nigerian rural small ruminant farmers' understanding of climate change. About $93 \%$ of the farmers claimed that they understand the concept of climate change. Most $(94.3 \%)$ of them stated that they had observed changes in climate parameters. The proportion of farmers who became aware of climate change through extension agents was low (10.3\%). This might not be unconnected with the dwindling number of extension agents in the Nation's Agricultural Development Projects (ADPs) as found by NAERLS and NFRA (2009). Personal experience was the most $(52.3 \%)$ prominent means through which they became aware of climate change. Only $4.0 \%$ of the rural farmers became aware of climate change concept through television broadcasts. 
Table 3: Distribution of respondents' understanding, awareness and sources of awareness of climate change

\begin{tabular}{|c|c|c|c|c|c|c|}
\hline \multirow[t]{2}{*}{ Variables } & \multicolumn{5}{|c|}{ State/Agro-ecological zone } & \multirow[t]{2}{*}{ Total } \\
\hline & $\begin{array}{l}\text { Kaduna- } \\
\text { North-west }\end{array}$ & $\begin{array}{l}\text { Niger-North- } \\
\text { central }\end{array}$ & $\begin{array}{l}\text { Borno-North- } \\
\text { east }\end{array}$ & $\begin{array}{l}\text { Oyo- } \\
\text { South- } \\
\text { west }\end{array}$ & $\begin{array}{l}\text { Abia- } \\
\text { Southeast }\end{array}$ & \\
\hline Awareness of climate change $(n=293)$ & $(n=60)$ & $(n=60)$ & $(n=55)$ & $(n=59)$ & $(n=59)$ & \\
\hline yes & $60(100)$ & $60(100)$ & $35(58.3)$ & $59(98.3)$ & 59 (98.3) & $273(91.0)$ \\
\hline no & $0(0.0)$ & $0(0.0)$ & $20(33.3)$ & $0(0.0)$ & $0(0.0)$ & $20(6.7)$ \\
\hline $\begin{array}{l}\text { Observed any form of change in } \\
\text { climate }(n=300)\end{array}$ & $(n=60)$ & $(n=60)$ & $(n=60)$ & $(\mathrm{n}=60)$ & $(n=60)$ & \\
\hline Yes & $57(95.0)$ & $57(95.0)$ & $52(86.7)$ & 59 (98.3) & $58(96.7)$ & $283(94.3)$ \\
\hline No. & $3(5.0)$ & $3(5.0)$ & $8(13.3)$ & $1(1.7)$ & $2(3.3)$ & $17(5.7)$ \\
\hline $\begin{array}{l}\text { Sources of awareness in change in } \\
\text { climate }(n=283)\end{array}$ & $(n=59)$ & $(n=60)$ & $(n=50)$ & $(n=54)$ & $(n=60)$ & \\
\hline Extension agent & $7(11.9)$ & $12(20)$ & $7(14)$ & $4(7.4)$ & $0(0.0)$ & $30(10.6)$ \\
\hline Radio & $6(10.2)$ & $3(5.0)$ & $14(28)$ & $29(53.7)$ & $22(36.7)$ & $74(26.1)$ \\
\hline Television & $3(5.1)$ & $1(1.7)$ & $0(0.0)$ & $7(12.9)$ & $0(0.0)$ & $11(3.9)$ \\
\hline Personal experience & $42(71.2)$ & $36(60)$ & $28(56)$ & $15(27.8)$ & $33(55.0)$ & $153(54.1)$ \\
\hline
\end{tabular}


Journal of Agricultural Extension Vol. 15 (2), December, 2011

Fellow farmers

$1(1.7)$

8(13.3)

$1(2.0)$

$0(0.0)$

$5(8.3)$

$15(5.3)$

Source: Field survey, 2010; Figures in parenthesis are percentage 


\section{Climate change parameters observed by farmers}

Climate change parameters observed by some of the farmers interviewed were shown in Table 4. Majority $(75.3 \%)$ of the small ruminant farmers reported that yearly rainfall ends late and about $68 \%$ reported increase in temperature. This increase in temperature might be in accordance with projections that average temperature could increase by another 1.4 to $5.8^{\circ} \mathrm{C}$ by 2100 (UNFCCC, 2005). Increased incidence of parasites was the most prominent observed change indicated by $49.3 \%$ of the respondents for goat and 38\% for sheep as shown in Table 5 . About 35\% of the respondents observed increase disease conditions and $33.7 \%$ mortality rate in sheep while $43.0 \%$ of the respondents observed increased disease condition and $40 \%$ reduced frequency of parturition ingoats. Also, reduction in appetite, growth rate as well as increased mortality and its frequency were other changes observed by respondents. Increase in temperature is capable of increasing the occurrence of animal diseases, as some species of insect serve as disease vectors, such as biting flies and ticks, are more likely to survive year-round. Certain existing parasitic diseases may also become more prevalent, or their geographical range may spread, if rainfall increases as reported by Epstein and Mills (2005). This may contribute to an increase in disease spread, including zoonotic diseases. 
Table 4: Climate Change Parameter Observed by small ruminant farmers

\begin{tabular}{|c|c|c|c|c|c|c|}
\hline \multirow[t]{2}{*}{ Variables } & \multicolumn{5}{|c|}{ State/Agro-ecological zone } & \multirow[t]{2}{*}{ Total } \\
\hline & $\begin{array}{l}\text { Kaduna- } \\
\text { North-west }\end{array}$ & $\begin{array}{l}\text { Niger- } \\
\text { North- } \\
\text { central }\end{array}$ & $\begin{array}{l}\text { Borno-North- } \\
\text { east }\end{array}$ & $\begin{array}{l}\text { Oyo-South- } \\
\text { west }\end{array}$ & $\begin{array}{l}\text { Abia- } \\
\text { Southeast }\end{array}$ & \\
\hline yearly rainfall begins early & $28(46.7)$ & $31(51.7)$ & $41(68.3)$ & $16(26.7)$ & $16(26.7)$ & $132(44.0)$ \\
\hline Yearly rainfall begins late & $56(93.3)$ & $40(66.7)$ & $13(21.7)$ & $42(70.0)$ & $54(90.0)$ & $205(68.3)$ \\
\hline Yearly rainfall ends early & $31(51.7)$ & $36(60.0)$ & $23(38.3)$ & $1(1.7)$ & $17(28.3)$ & $108(36.0)$ \\
\hline Yearly rainfall end late & $55(91.7)$ & $28(46.7)$ & $33(55.0)$ & $56(93.3)$ & $54(90.0)$ & $226(75.3)$ \\
\hline Increase in temperature & $56(93.3)$ & $40(66.7)$ & $34(56.7)$ & $27(45.0)$ & $48(80.0)$ & $205(68.3)$ \\
\hline Increased coldness & $31(51.7)$ & $39(65.0)$ & $28(46.7)$ & $11(18.3)$ & $23(38.3)$ & $132(44.0)$ \\
\hline Early dryness of pasture & $54(90.0)$ & $38(63.3)$ & $41(68.3)$ & $1(1.7)$ & $8(13.3)$ & $142(47.3)$ \\
\hline Early dryness of water sources & $52(86.7)$ & $33(55.0)$ & $30(50.0)$ & $1(1.7)$ & $9(15.0)$ & $125(41.8)$ \\
\hline
\end{tabular}

Source: Field survey, 2010; Figures in parenthesis are percentage 


\section{Frequency of observed changes in small ruminants probably attributed to climate} change

Table 5 shows the frequency of observed changes in small ruminants probably due to climate change.Majority $(38.0 \%)$ of the farmers observed increased incidence of parasites on sheep and goats $(49.3 \%)$. Also $43 \%$ of the respondents observed increased disease condition in goats and $33.7 \%$ increased mortality in sheeps.

Table 5: Frequency of observed changes in small ruminants attributed to climate change $(n=300)$

\begin{tabular}{|c|c|c|c|c|c|c|c|c|c|c|c|c|}
\hline \multirow{3}{*}{$\begin{array}{l}\text { Observed } \\
\text { changes }\end{array}$} & \multicolumn{10}{|c|}{ State/Agro-ecological zone } & \multicolumn{2}{|c|}{ Total } \\
\hline & \multicolumn{2}{|c|}{$\begin{array}{l}\text { Kaduna- } \\
\text { North-west }\end{array}$} & \multicolumn{2}{|c|}{$\begin{array}{l}\text { Niger-North- } \\
\text { central }\end{array}$} & \multicolumn{2}{|c|}{$\begin{array}{l}\text { Borno-North- } \\
\text { east }\end{array}$} & \multicolumn{2}{|c|}{$\begin{array}{l}\text { Oyo-South- } \\
\text { west }\end{array}$} & \multicolumn{2}{|c|}{ Abia-Southeast } & \multirow[b]{2}{*}{ sheep } & \multirow[b]{2}{*}{ Goat } \\
\hline & sheep & goat & sheep & goat & sheep & goat & sheep & goat & sheep & Goat & & \\
\hline $\begin{array}{l}\text { Reduced } \\
\text { appetite }\end{array}$ & $\begin{array}{l}44 \\
(73.3)\end{array}$ & $\begin{array}{l}37 \\
(61.7)\end{array}$ & $\begin{array}{l}22 \\
(36.7)\end{array}$ & $\begin{array}{l}29 \\
(48.3)\end{array}$ & $\begin{array}{l}18 \\
(30.0)\end{array}$ & $\begin{array}{l}11 \\
(18.3)\end{array}$ & $\begin{array}{l}2 \\
(3.3)\end{array}$ & $\begin{array}{l}6 \\
(10.0)\end{array}$ & $\begin{array}{l}2 \\
(3.3)\end{array}$ & $7(11.7)$ & $\begin{array}{l}88 \\
(29.3)\end{array}$ & $\begin{array}{l}90 \\
(30.0)\end{array}$ \\
\hline $\begin{array}{l}\text { Increased } \\
\text { appetite }\end{array}$ & $\begin{array}{l}16 \\
(26.7)\end{array}$ & $9(15.0)$ & $\begin{array}{l}13 \\
(21.7)\end{array}$ & $18(30.0)$ & $\begin{array}{l}34 \\
(56.7)\end{array}$ & $30(50.0)$ & $\begin{array}{l}6 \\
(10.0)\end{array}$ & $\begin{array}{l}13 \\
(21.7)\end{array}$ & $\begin{array}{l}8 \\
(13.3)\end{array}$ & $43(71.7)$ & $\begin{array}{l}77 \\
(25.7\end{array}$ & $\begin{array}{l}113 \\
(37.7)\end{array}$ \\
\hline $\begin{array}{l}\text { Reduced } \\
\text { frequency } \\
\text { of birth }\end{array}$ & $\begin{array}{l}42 \\
(70.0)\end{array}$ & $\begin{array}{l}35 \\
(58.3)\end{array}$ & $\begin{array}{l}24 \\
(40.0)\end{array}$ & $\begin{array}{l}29 \\
(48.3)\end{array}$ & $\begin{array}{l}17 \\
(28.3)\end{array}$ & $\begin{array}{l}11 \\
(18.3)\end{array}$ & $\begin{array}{l}9 \\
(15.0)\end{array}$ & $\begin{array}{l}23 \\
(38.3)\end{array}$ & $\begin{array}{l}3 \\
(5.0)\end{array}$ & $\begin{array}{l}22 \\
(36.7)\end{array}$ & $\begin{array}{l}95 \\
(31.7)\end{array}$ & $\begin{array}{l}120 \\
(40.0)\end{array}$ \\
\hline $\begin{array}{l}\text { Increased } \\
\text { frequency } \\
\text { of birth }\end{array}$ & $\begin{array}{l}12 \\
(20)\end{array}$ & $4(6.70$ & $\begin{array}{l}8 \\
(13.3)\end{array}$ & $\begin{array}{l}16 \\
(26.7)\end{array}$ & $\begin{array}{l}32 \\
(53.3)\end{array}$ & $\begin{array}{l}25 \\
(41.7)\end{array}$ & $\begin{array}{l}1 \\
(16.7)\end{array}$ & $\begin{array}{l}3 \\
(5.0)\end{array}$ & $\begin{array}{l}6 \\
(10.0)\end{array}$ & $\begin{array}{l}22 \\
(36.7)\end{array}$ & $\begin{array}{l}59 \\
(19.7)\end{array}$ & $\begin{array}{l}70 \\
(23.3)\end{array}$ \\
\hline $\begin{array}{l}\text { Reduced } \\
\text { growth } \\
\text { rate }\end{array}$ & $\begin{array}{l}44 \\
(73.3)\end{array}$ & $\begin{array}{l}39 \\
(65.0)\end{array}$ & $\begin{array}{l}22 \\
(36.7)\end{array}$ & $\begin{array}{l}28 \\
(46.7)\end{array}$ & $\begin{array}{l}21 \\
(35.0)\end{array}$ & $\begin{array}{l}11 \\
(18.3)\end{array}$ & $\begin{array}{l}11 \\
(18.3)\end{array}$ & $\begin{array}{l}24 \\
(40.0)\end{array}$ & $\begin{array}{l}2 \\
(3.3)\end{array}$ & $\begin{array}{l}14 \\
(23.3)\end{array}$ & $\begin{array}{l}100 \\
(33.3)\end{array}$ & $\begin{array}{l}116 \\
(38.7)\end{array}$ \\
\hline $\begin{array}{l}\text { Increased } \\
\text { growth }\end{array}$ & 13 & 6 & 13 & 22 & 29 & 26 & 2 & 11 & 10 & 34 & 67 & 99 \\
\hline
\end{tabular}


Journal of Agricultural Extension

Vol. 15 (2), December, 2011

rate

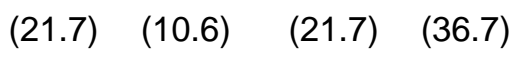

(48.3) (43.3)

(3.3)

(18.3) (16.7) (56.7)

$(22.4) \quad(33.0)$

Increased $\quad 46 \quad 38 \quad 24 \quad 34$

$26 \quad 16$

1342

18

incidence (76.7) (63.3)

(40) (56.7)

(43.3) (26.7)

$\begin{array}{llll}(21.7) & (70.0) & (8.3) \quad(30.0)\end{array}$

114148

of

parasites

of

Decreased 14

$14 \quad 10$

incidence

parasites

$19 \quad 19$

(31.7) (31.7)

(16.7) (18.3)

(23.3) (16.7)

$\begin{array}{lllllllllllll}\text { Increased } & 42 & 37 & 26 & 28 & 25 & 14 & 10 & 37 & 3 & 13 & 106 & 129 \\ \text { disease } & (70) & (61.7) & (43.3) & (46.7) & (41.7) & (23.3) & (16.7) & (61.7) & (5.0) & (21.7) & (35.3) & (34.0)\end{array}$

conditions

$\begin{array}{lllll}\text { Decreased } & 14 & 7 & 10 & 18\end{array}$

disease

conditions
23(38.3) 2

(3.3)
3
$(5.0)$

6

(10.0)
3

(5.0)
22

(36.7)
49

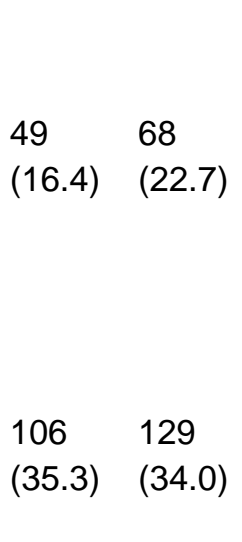




\section{Frequency of adaptive measures used by rural small ruminant farmers:}

The frequency of adaptive methods employed by small ruminants farmers is presented in Table 6. Ninety percent $(90 \%)$ of the farmers indicated that they used provision of of housing for their animals while $77 \%$ used provision of shade for animals during the day as an adaptive measure. Over $80 \%$ used keeping and feeding the animals during the rains and cold as a measure. These are normal husbandry practices employed by the farmers

Table 6: Freqency of adaptive methods used by rural small ruminant farmers $(n=300)$

\begin{tabular}{|c|c|c|c|c|c|c|}
\hline \multirow[t]{2}{*}{ Variables } & \multicolumn{5}{|c|}{ State/Agro-ecological zone } & \multirow[t]{2}{*}{ Total } \\
\hline & $\begin{array}{l}\text { Kaduna- } \\
\text { North-west }\end{array}$ & $\begin{array}{l}\text { Niger- } \\
\text { North- } \\
\text { central }\end{array}$ & $\begin{array}{l}\text { Borno- } \\
\text { North- } \\
\text { east }\end{array}$ & $\begin{array}{l}\text { Oyo- } \\
\text { South- } \\
\text { west }\end{array}$ & $\begin{array}{l}\text { Abia- } \\
\text { South } \\
\text { East }\end{array}$ & \\
\hline Provision of housing for the animals & $58(96.7)$ & $58(96.7)$ & $52(86.7)$ & $48(80)$ & $54(90)$ & $270(90)$ \\
\hline Frequent cleaning of the house & $57(95.0)$ & $60(100)$ & $54(90)$ & $50(83.3)$ & $54(90)$ & $275(91.7)$ \\
\hline $\begin{array}{l}\text { Good health care by inviting vet } \\
\text { doctor }\end{array}$ & $59(98.3)$ & $58(96.7)$ & $50(83.3)$ & $42(70)$. & $6(10)$ & $215(71.7)$ \\
\hline $\begin{array}{l}\text { Provision of shade for animals during } \\
\text { the day }\end{array}$ & $53(88.3)$ & $43(71.7)$ & $43(71.7)$ & $51(85)$ & $41(68.3)$ & $231(77)$ \\
\hline Traditional health care & $47(78.3)$ & $47(78.3)$ & $24(40.0)$ & $28(46.7)$ & $40(66.7)$ & $186(62)$ \\
\hline Good feed and water provision & $60(100)$ & $49(81.7)$ & $52(86.7)$ & $51(85.0)$ & $44(73.3)$ & $256(85.3)$ \\
\hline Provision of feed supplements & $49(81.7)$ & $39(65.0)$ & $36(60.0)$ & $56(93.3)$ & $27(45.0)$ & 207 (69) \\
\hline $\begin{array}{l}\text { Control of internal and external } \\
\text { parasites }\end{array}$ & $58(96.7)$ & $41(68.3)$ & $43(71.7)$ & $43(71.7)$ & $31(51.7)$ & $216(72)$ \\
\hline $\begin{array}{l}\text { Keeping and feeding the animals } \\
\text { during rainfall and cold }\end{array}$ & $53(88.3)$ & $48(80.0)$ & $45(75.0)$ & $46(76.7)$ & $50(83.3)$ & $242(80.7)$ \\
\hline Irrigation of pasture during dry & $40(66.7)$ & $26(43.3)$ & $30(50.0)$ & $1(1.7)$ & $17(28.3)$ & 114 (38.) \\
\hline
\end{tabular}




\section{Percieved rating of effectiveness of adaptive methods used by rural small ruminant farmers}

Table 7 shows percieved rating of effectiveness of adaptive methods used by rural small ruminant farmers most of the adopted adaptive strategies were effective. For example, over $87 \%$ believe that provision of housing for the animals is effective inprobably combating the negative effect of climate change on their sheep and goats. The weighted average for traditional health care (mean=2.2) and irrigation of pasture during dry season (mean=2.0) were below the mean of Likert scale (mean=2.5), therefore the adaptive measures indicated thatwere not effective in probably combating the effect of climate change on a general note

Table 7: Percieved rating of effectiveness of adaptive methods used by rural small ruminant farmers $(n=300)$

\begin{tabular}{|c|c|c|c|c|c|c|c|}
\hline \multirow[b]{2}{*}{ Adaptive Strategies } & \multicolumn{5}{|c|}{ State/Agro-ecological zone } & \multirow{2}{*}{$\begin{array}{l}\text { Over all } \\
\text { mean }\end{array}$} & \multirow{2}{*}{$\begin{array}{l}\text { Overall } \\
\text { percep } \\
\text { tion }\end{array}$} \\
\hline & $\begin{array}{l}\text { Kaduna- } \\
\text { North-west }\end{array}$ & $\begin{array}{l}\text { Niger- } \\
\text { North- } \\
\text { central }\end{array}$ & $\begin{array}{l}\text { Borno- } \\
\text { North- } \\
\text { east }\end{array}$ & $\begin{array}{l}\text { Oyo- } \\
\text { South- } \\
\text { west }\end{array}$ & $\begin{array}{l}\text { Abia- } \\
\text { Southeas } \\
t\end{array}$ & & \\
\hline $\begin{array}{l}\text { Provision of housing for the } \\
\text { animals }\end{array}$ & 3.7 & 3.2 & 2.8 & 3.6 & 3.8 & 3.3 & $\begin{array}{l}\text { effectiv } \\
\text { e }\end{array}$ \\
\hline Frequent cleaning of the house & 3.8 & 3.9 & 2.9 & 3.6 & 3.2 & 3.4 & $\begin{array}{l}\text { effectiv } \\
\text { e }\end{array}$ \\
\hline $\begin{array}{l}\text { Good health care by inviting vet } \\
\text { doctor }\end{array}$ & 3.8 & 3.4 & 3.1 & 3.5 & 1.2 & 3.1 & $\begin{array}{l}\text { effectiv } \\
\text { e }\end{array}$ \\
\hline $\begin{array}{l}\text { Provision of shade for animals } \\
\text { during the day }\end{array}$ & 3.7 & 3.0 & 2.8 & 3.5 & 3.0 & 3.2 & $\begin{array}{l}\text { effectiv } \\
\text { e }\end{array}$ \\
\hline \multirow[t]{2}{*}{ Traditional health care } & & & & & & & not \\
\hline & 2.2 & 2.5 & 2.0 & 2.8 & 2.3 & 2.4 & $\begin{array}{l}\text { effectiv } \\
\text { e }\end{array}$ \\
\hline Good feed and water provision & 3.8 & 3.4 & 3.1 & 3.8 & 3.4 & 3.5 & $\begin{array}{l}\text { effectiv } \\
\text { e }\end{array}$ \\
\hline Provision of feed supplements & 3.8 & 3.1 & 2.9 & 3.7 & 2.9 & 3.3 & $\begin{array}{l}\text { effectiv } \\
\text { e }\end{array}$ \\
\hline Control of internal and external & 3.8 & 3.0 & 3.0 & 3.6 & 2.4 & 3.2 & effectiv \\
\hline
\end{tabular}


parasites

e

Keeping and feeding the animals indoor during rainfall 3.8 and cold/harmatan

Irrigation of pasture during dry season

\section{8}

2.6

2.9

3.7

3.1

3.2

effectiv

e

2.0

2.3

2.0

2.0

2.2

effectiv

e

Source: Field survey, 2010; Figures are weighted mean of scale of 1 to 4 (very effective to not effective).

\section{Conclusion and recommendations}

It is clear from this study that majority of small ruminant farmers in the rural Nigeria are aware of what climate change is all about and many of them have observed changes in climage through personal experience.Majority of them have also observed and seems to also attributed changes to effect of climate change on their flock. Majority of them have also used various methods which are husbandry practices to combat climate change.They are however aware that traditional health care and irrigation of pasture during dry season are not effective adaptive methods to combat climate change. There is need to educate the farmers more on the possible effect of climate change through the use of radio and other extension organizations (government and non-governmental) on the current situation and implication of climate change on themselves and their animals.

\section{References}

Ajala, M.K., Lamidi, O.S. and Otaru, S.M. (2010). Peri-urban small ruminant production in Northern guinea savanna, Nigeria. Asian Journal of Animal and Veterinary Advances, 3(3): 138-146.

Technical Centre for Agricultural and Rural Co-operation (CTA)(2009). Implication of climate change for sustainable agricultural production systems in ACP countries: Getting information and communication strategies right.A Compilation Document

Deressa , T.T. and R.M. Hassan (2009) Economic impact of climate change on crop production in Ethiopia: Evidence from cross-section measuers. Journal of African Economies. Vol 18 (4) pp.529-554. 
Epstein and Mills (2005).Climate change future: Health, ecological and economical dimensions. The Centre for Health and the Global Environment.

FAO (2009). Climate change in Africa: The threat to agriculture.http//www.fao.org.africa. 10th January,2010.

IPCC (2007). Summary for policymakers. In: Climate change 2007: impacts, adaptation and vulnerability. Contribution of working group ii to the fourth assessment report of the Intergovernmental Panel on Climate Change (M.L. Parry et al. (eds.)). Cambridge University Press, Cambridge, U.K., and New York, N.Y., U.S.A.. pp. 7-22. http://www.ipcc.ch/publications and data/publications and data reports.htm. Retrieved 2009-05-20.

International Food Policy Research Institute (2009). Climate change: Impact on agriculture and costs of adaptation. http://www.ifpri.org/publication. 10th january, 2010

lyiola-Tunji, A. O. and F. O. Issa (2010). Small ruminant production characteristics by rural farm families in NAERLS-adopted and non-adopted villages in North-Western Nigeria. Continental Journal of Animal and Veterinary Research, 2: 18 - 24.

Kurukulsuriya, P and R. Mendelson ( 2007). Endogenous irrigation: The impact of climate change on farmers in Africa. World Bank Policy Research Working Paper 427

NAERLS and NFRA (2009): Annual Agricultural Performance Survey Report of Nigeria: 2009 Wet Season. NAERLS Press, pp. 134.

UNFCCC. (2005). Feeling the heat. Electronic background document - united nations framework convention on climate change, (http://unfccc.int/essential background/feeling the heat/items/2918.php).

Watson R.T, Zinyowera. M.C and R.H. Moses (1997). The regional impact of climate change: An assessment of vulnerable panel on climate change. http://www.ipcc.ch. 10th January, 2010 